\title{
Primary Immunodeficiency Diseases in Iran: Past, Present and Future
}

\author{
Asghar Aghamohammadi, MD, PhD ${ }^{1,2}$; Hassan Abolhassani, MD, PhD ${ }^{1,2}$; Nima Rezaei, MD, PhD ${ }^{1,2,3^{*}}$ \\ ${ }^{1}$ Research Center for Immunodeficiencies, Pediatrics Center of Excellence, Children's Medical Center, Tehran University of Medical Sciences, \\ Tehran, Iran \\ ${ }^{2}$ Primary Immunodeficiency Diseases Network (PIDNet), Universal Scientific Education and Research Network (USERN), Tehran, Iran \\ ${ }^{3}$ Department of Immunology, School of Medicine, Tehran University of Medical Sciences, Tehran, Iran
}

\begin{abstract}
Clinical immunology and its subset topics are rather newly emerging medical fields in Iran as well as other developing countries. Primary immunodeficiency diagnosis and treatment were revolutionized in the late 1970s; a period of time that coincided with the establishment of the Division of Clinical Immunology and Allergy at the Children's Medical Center, Tehran. Subsequently, the launch of fellowship training programs (in 1988), the development of a national Iranian Primary Immunodeficiency Diseases Registry (in 1999), the inauguration of Research Center for Immunodeficiencies (in 2009), and recently, the national primary immunodeficiency network (in 2016) significantly changed the picture of disease management during the last 40 years. In this review, we seek to elucidate the most important past events, current challenges and future directions regarding the field of primary immunodeficiency.

Keywords: Clinical immunology, Diagnosis, Immunodeficiency Diseases, Iran

Cite this article as: Aghamohammadi A, Abolhassani H, Rezaei N. Primary immunodeficiency diseases in Iran: past, present and future. Arch Iran Med. 2021;24(2):118-124. doi: 10.34172/aim.2021.18.
\end{abstract}

Received: January 9, 2019, Accepted: May 20, 2020, ePublished: February 1, 2021

\section{Introduction}

Primary immunodeficiency diseases (PID) constitute a group of approximately 400 inherited disorders caused by qualitative or quantitative defects of the immune system components. ${ }^{1}$ Depending on the defective component and etiologies, the affected patients can present with increased susceptibility to infections or other non-infectious manifestations including autoimmunity, immune dysregulation, allergic diseases, lymphoproliferation, and cancer. ${ }^{2}$ Of note, the history of clinical PID patients dates back to a report by Zakariya al-Razi (880-932 A.D) about the higher susceptibilities of some individuals to smallpox and measles compared to the public. ${ }^{3}$ However, the field of PID has been re-considered after the development of broad-spectrum antibiotics and preventive vaccines when physicians observed that despite these medical modalities, the severity of medical condition leads to morbidity and mortality in a selected group of patients. ${ }^{4}$

Recently, we have published several studies and reviews regarding the evidence demonstrating that Iran, as a leading country in the region of the Middle East, has made appropriate progress in both basic and clinical immunology, and described the influences of those achievements in the specific field of PIDs in Iran. ${ }^{4,5}$ This review aims to summarize those reports to highlight the strengths and weaknesses and, at the same time, address the expected challenges and opportunities in the future.

\section{Progression of Primary Immunodeficiency Field}

Improvements began when Professor Abolhasan Farhoudi (1924-2006), an academic member of Tehran University of Medical Sciences (TUMS), decided to launch the Division of Clinical Immunology and Allergy complemented by an Immunology Laboratory. The division and the laboratory were founded at Children's Medical Center. ${ }^{4}$ This step significantly changed the diagnosis and treatment of patients with PID by designing targeted medical archiving, family history taking and providing a sustainable treating unit for diagnosed patients. ${ }^{4}$ The next pioneering step was the evaluation of PID frequency at the national level which was performed by trained clinical immunologists of TUMS and their medical students in $1998 .^{6}$ Their efforts toward their main goals lead to the establishment of a national registry which aimed not only to identify the national frequency of various PIDs entities, but also to improve the therapeutic modalities of patients with longterm follow-up, documentation of natural history and consequential complications experienced by patients in different organs, and subsequently to improve progressive molecular/clinical research in the field of PIDs in Iran. ${ }^{4,5}$

After five years of endeavors, the first data collected in the Iranian PID registry was published in 2002, including 
440 PID patients within the four main categories of the disease. Recently, we have published the 20-year survey of the PID registry from the recently structured national PID network organizing 31 collaborating hospitals affiliated to 26 medical science universities from the main provinces. ${ }^{7-10}$ Now, we have registered 3056 (39.3\% female) and with molecular diagnosis confirmed in 1014 patients (33.1\%). Although this report magnifies the magnificent improvement of expert training and increased awareness of targeted physicians (point prevalence of 1/26000), $7,11,12$ we estimate a prevalence of $1 / 600$ for PIDs in Iran due to a higher rate of consanguinity in the country compared to Western countries. In other words, we expect more than 130000 undiagnosed patients based on the prevalence of PIDs in Iran; therefore, the current number of clinically registered PID patients (2.3\% of expected patients) indicates a long way to go and further required supports to accomplish the task. So far, definite diagnosis, assigned by molecular confirmation, has been made in $33.1 \%$ of the total Iranian PID cases which represents an equal rate to the frequency of genetic diagnosis in Western countries with long-term established PID registries. ${ }^{7}$ Facing the therapeutic issues of registered patients and an increasing number of cases who need sustainable treatment, the Iranian Primary immunodeficiency Association (Iranian PiA) was established. This initiative led to an agreement with the Ministry of Health for financial support to cover up to $80 \%$ of immunoglobulin treatment costs.

The majority of these advanced outcomes became possible due to the focused activity of a dedicated research center, Research Center for Immunodeficiencies (RCID, http://rcid.tums.ac.ir/). Based on the commitment delivered by the Non-Communicable Diseases Center of the Ministry of Health, RCID was launched as a tertiary center for the molecular diagnosis of immunodeficiency diseases in Iran. Routine referrals of PID patients from peripheral centers to RCID are made for the performance of advanced immunologic tests and molecular diagnosis. ${ }^{13-15}$ The European Society for Immunodeficiencies (ESID) has currently accepted RCID as the only documenting center from Iran to update the epidemiological PID indexes from the country (https://esid.org/Working-Parties/RegistryWorking-Party/Documenting-centers/AIR-IranianPrimary-Immunodeficiency-Registry-IPIDR). The Jeffrey Modell Foundation, which documents the global rate of PID, denotes RCID as the only Iranian center reporting its annual PID registry update among 358 institutions from 86 countries spanning 6 continents (https://www. info4pi.org). ${ }^{5}$

RCID, through a progressive foundation of different PID entity working parties, facilitates basic and advance research with the Iranian PID network for all collaborative parties and also provides unique materials and opportunities for international collaboration (http://ipin.tums.ac.ir/). ${ }^{5}$ The structure of the network constitutes more than 60 main clinical immunologists and subspecialists from the 20 main treating PID centers of the country. Accordingly, since the beginning of the 21st century, more than 350 publications in the field of PIDs have been indexed in major medical journals in the field of immunology showing our significant advancement by standing among the top 15 countries in the field (Figure 1). Approximately $50 \%$ of these publications are the outcome of international collaborations with advanced PID centers across the world (http://isid.research.ac.ir/). Of note, the national PID registry has had a great impact on novel PID gene discovery including finding mutations in the HAX1, ${ }^{16}$ G6PC $3,{ }^{17}$ ELA2, ${ }^{18}$ JAGN $1,{ }^{19}$ CARD $9,{ }^{20}$ STK $4,{ }^{21}$ $L R B A{ }^{22}$ and $C D 70$ genes. ${ }^{23}$

During the last decade, several other research centers have also contributed to some aspects of PID in Iran. Of note, the immunology, asthma and allergy institute of TUMS was dedicated to setting up some advanced laboratory tests regarding specific phagocytosis and combined immunodeficiency diseases. Moreover, they have performed several studies toward the facilitation of hematopoietic stem cell transplantation and newborn screening of the patients. Other main centers active in the national network in term of patient identification and laboratory services include Non-communicable Diseases Research Center (Alborz University of Medical Sciences),

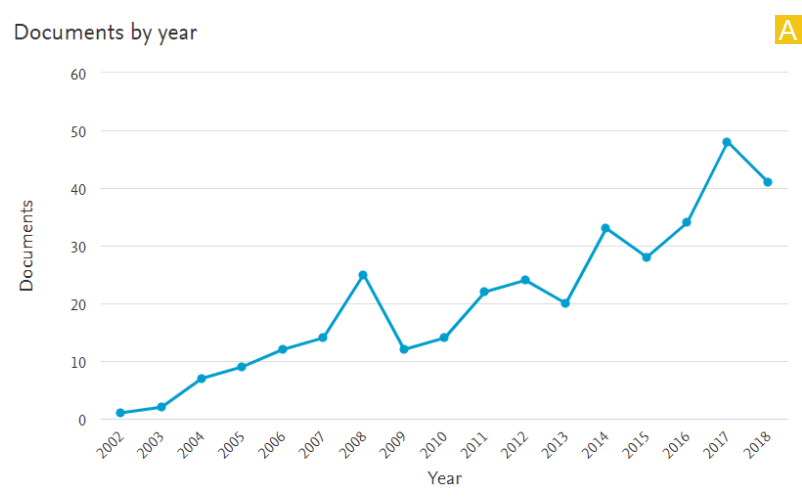

Documents by country or territory

Compare the document counts for up to 15 countries/territories

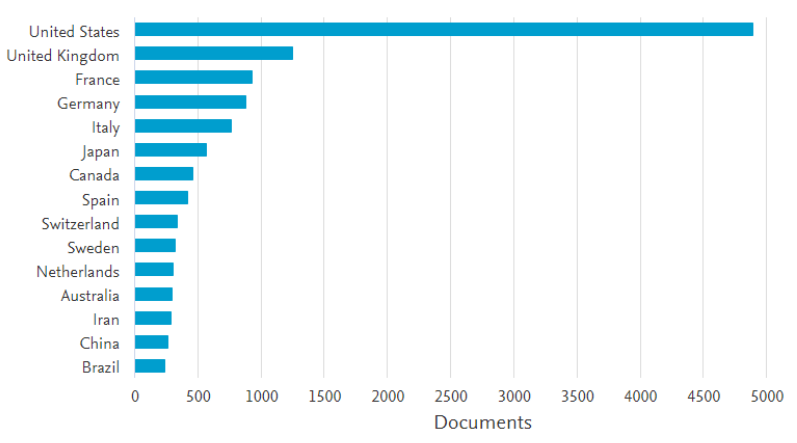

Figure 1. Scientific Output of Iran in the Field of Primary Immunodeficiency Diseases. A) Scientifc output of the country during last 20 years $(n=347$ docuemnts). B) The ranking of 15 top countries with the highest scientific output in this field. 
Pediatric Infections Research Center (Mofid Children's Hospital, Shahid Beheshti University of Medical Sciences), Pediatric Respiratory Diseases Research Center, National Research Institute of Tuberculosis and Lung Diseases (Shahid Beheshti University of Medical Sciences), Child Growth and Development Research Center, Research Institute for Primordial Prevention of Non-Communicable Disease (Isfahan University of Medical Sciences,), Hematology, Oncology and Stem Cell Transplantation Research Center (Tehran University of Medical Sciences), Acquired Immunodeficiency Research Center (Isfahan University of Medical Sciences), Allergy Research Center (Shiraz University of Medical Sciences), Allergy Research Center (Mashhad University of Medical Sciences), Noncommunicable Pediatric Diseases Research Center (Babol University of Medical Sciences) and Pediatric Infectious Diseases Research Center ( Mazandaran University of Medical Sciences).

RCID continuously follows the process of education and meetings on PID by organizing an annual international congress of PID (ICID) during World PID Week (WPIW) in Iran. ${ }^{5,11,12}$ This research center is also a sister of J-Project, entitled J-Persia, to take Iran as a pioneer country in Central Asia to expand and accelerate its essential impact in Persian-speaking countries (particularly Afghanistan and Tajikistan, http://www.jprojectnetwork.com/).

Continuing medical education programs for targeted physicians in different provinces, ${ }^{5}$ launching "Ph.D. by research" and "clinician-researchers" training programs in the field of primary immunodeficiency, ${ }^{5}$ publication of several international PID textbooks and guidelines, ${ }^{24-36}$ and having three PID researchers honored as the top 1\% of the most-cited scientists in the category of immunology according to Thomson Scientific's Essential Science Indicators (ESI, https://clarivate.com) are among other major achievements during the last decade. Publication of three specialized journals of "Immunology and Genetics Journal” (https://igj.tums.ac.ir/index.php/igj), "Iranian Journal of Immunology" (http://iji.sums.ac.ir/) and Iranian Journal of Allergy, Asthma and Immunology (http://ijaai.tums.ac.ir/index.php/ijaai) has contributed to the visibility of PID research outcomes internationally. For details of these outcomes, please refer to the recent review published about the current status of PID in Iran. ${ }^{5}$

\section{Current Achievements and Obstacles in the Primary Immunodeficiency Field}

The above-mentioned national activities and international collaborations have led to several current achievements about the practical aspects of diagnosis and management of patients with both well-known and newly described PID. Establishment and continued efforts for registration of clinical and immunologic profiles of patients improved genetic testing to guide both definitive diagnosis and decision making. ${ }^{15,37}$
With a current multidisciplinary approach to the diagnosis of PID, our national registry was involved in the identification of complicated immune dysregulation and unusual presentations of the diseases. ${ }^{5}$ These findings enabled us to participate in novel gene discovery and improved targeted therapies through an understanding of the mechanisms that underlie these events. ${ }^{38-40}$ By generation of comprehensive national networks and specific PID group working parties, we collected the best practices in the management of PID entities from an expert team as a consensus guideline. ${ }^{41}$ This guideline addresses basic and advanced immune function evaluation and practical advice for physicians regarding vaccination and treatment adjustment using available new products. ${ }^{42-46}$

The improved survival rate of PID patients and lateonset presentation of the hypomorphic phenotypes urged us to not confine our practice to pediatric patients and address the increasing number of more adults with immune defects. ${ }^{47-49}$ The diagnostic workup of adult patients with suspected PID is more comprehensive by taking a detailed patient and family history, immunological and laboratory assays that would be followed by the next step, targeted or next-generation sequencing. These novel approaches for the identification of underlying PID-causing gene defects in both pediatric and adult patients have enabled rapid and cost-effective diagnosis and correct treatment. ${ }^{15,33}$

With a combination of long-term follow-up and molecular advanced technology, we now have participated in the extension of the phenotypes of previously reported known PID genes presenting with distinct manifestations from the original description. ${ }^{15,48,50-53}$ However, we need further improvement regarding the scaling of the sequencing costs and pipeline time to provide faster and appropriate management for all patients nationwide.

Allogeneic hematopoietic stem cell transplantation remains the main curative therapy for most severe PIDs and its application and patients who require this modality are increasing, related to better genetic diagnosis in the patients. ${ }^{50}$ Despite the presence of several studies reporting improved outcomes in PID patients who undergo transplantation, this treatment can be offered to only less than $5 \%$ of indicated patients in our registry due to costs, inappropriate infrastructure and difficulties in HLA-match donor selection. ${ }^{5}$ To tackle this limitation, we should punctually improve our experience in hematopoietic stem cell transplantation for PIDs to fulfill criteria that have been mentioned by the Primary Immune Deficiency Treatment Consortium.

Since immunoglobulin replacement is an essential component in the treatment of different types of PID affecting antibody production, sustainability and appropriate preparation of this modality were targeted by our national efforts. For all PIDs needing immunoglobulin replacement, standardized and practical guidelines have been defined for administration in hospital settings. ${ }^{54-56}$ 
Regarding the remaining obstacles for replacement therapy, improved education (about the therapeutic modality) is an immediate need for patients and their families prior to the first course of therapy. By improving practices regarding pretreatment conditioning, immunoglobulin therapy should be improved by the availability of different commercial formulations including more concentrated solutions, more subcutaneous injection routes and more home-based care. ${ }^{24,56}$ The results of many different Western PID registry patients with complete modern modalities and biological agents for novel applications in patients with PIDs of treatment enforce the necessary activities toward development or importation of different modalities for our patients in the current routine including experience of thymus transplantation and other antagonist/agonist monoclonal antibodies.

Certain concerns still should be addressed for the current status in basic and clinical immunology education by revising study systems and residency programs, but efforts are ongoing with generating more postgraduate courses and fellowships to provide information and courses, especially on immunological diseases. ${ }^{5}$ Currently, almost 20 educational centers (in 17 cities including Ahvaz, Arak, Tehran, Isfahan, Babol, Birjand, Tabriz, Shaherkord, Shiraz, Semnan, Sanandaj, Kerman, Sari, Kermanshah, Hamedan, Mashhad and Yazd) train on the average 200 new basic immunologists in Master of science or $\mathrm{PhD}$ levels per year which sufficiently provide the need for human resources in this field. Although we have moved far from the poor conditions we had across the country to increase awareness about PID during the last decade, ${ }^{11,12}$ continuing education and training schedules are needed for pediatricians and general practitioners, as well as for specialists, to ensure that they have the same access to required information regarding diagnosis and the referring system. ${ }^{7,33}$ These efforts would improve early diagnosis and promote referrals to immunologists by the continuous support of the annual conference in the capital and peripheral symposia or summer schools for junior immunologists to help create networks of trained immunologists, and ensure updated information about PID.

\section{Future Direction and Strategy in Primary Immunodeficiency Field}

Personalized therapies and treatments of PID are the ultimate goals for clinical immunologists that should be provided for the patients in our national registry, as well. ${ }^{15,50}$ Characterization of the molecular defect and its affected signaling in undiagnosed patients with complicated diagnosis due to digenic or polygenic features should be addressed in near future to selectively add with adjuvant drugs or disease-modifying agents. ${ }^{7,15,37,57}$ Even the interaction of epigenetic factors and environmental parameters should be studied to depict the mysteries behind this group of PID patients. ${ }^{15}$

Although we are still struggling with standardization and the expansion of the newborn severe combined immunodeficiency and antibody deficiency screening, ${ }^{50}$ we should also move forward for pre-symptomatic diagnosis of patients with other types of PIDs, including phagocytosis and complement deficiency. The current promising results of the newborn screening program have been highly efficient in the detection of affected neonates and therefore, we observed an increase in the rate of referrals for transplantation and earlier medical interventions, which have improved life-expectancy in these patients. However, with the current capacity in transplantation, we should be also ready to deal with the increasing number of requests and prioritization of the process as well as turn-over of reporting and follow-up confirmation of the preliminary positive results. ${ }^{50}$

As we published our current activities for strong consensus recommendations towards the use of our genetic findings in managing patients with PID for prenatal diagnosis and treatment, ${ }^{7,15,50}$ future guidance for the use of genetic testing in PIDs should be completed by preimplantation genetic diagnosis and justifying the economic aspects of the right access of all patients and their families to these facilities.

Although we have estimated the burden of frequently diagnosed PIDs and the direct and indirect costs representing a heavy burden of disease on the patient and society, ${ }^{58-60}$ the process should continue to provide feedback to health care providers and policymakers regarding the decision for the administration of new agents or modalities for these patients.

We hope that the future will bring spectacular advances compared to the past decades and by current promising strengths, ${ }^{2}$ one can only imagine that hundreds of clinicians and basic scientists provide human and laboratory facilities to discover the different dimensions of PID that will affect therapeutic interventions. Perhaps the distinctions between primary and secondary immune effects will become blurred as we will discover the correct interaction of internal risks and external insults that cause susceptibility of the host to specific pathogens. We will apply in silico modeling and experimental assays to identify both key methodologies for the improvement of our knowledge regarding the pathophysiology of the novel PID diseases and related targeted medical action, and in the design of immune pathway studies. Given the complexities of our future concept of PID, there will be an ever-increasing need for better treatment paradigms regarding other common diseases including cancer, allergy, and autoimmunity.

Ultimately, better insights on the underlying genetic and non-genetic factors of PID may help to understand the pathogenesis and predict the clinical prognosis, resulting in both advanced management of the PID- 
associated complications and moving toward integration of personalized therapies for the highest quality of life. ${ }^{61-}$ ${ }^{63}$ Systematic approaches within the field of PID and an established national PID network consisting of clinicians and basic scientists in the field will help us to reinforce all scientific progress and human resources within the countries to discover a better solution for current general and regional PID challenges.

\section{Authors' Contribution}

AA and NR: The conception and design of the study, interpretation of data, revising it critically for important intellectual content and final approval of the version to be submitted. HA: Acquisition and interpretation of data, drafting the article and final approval of the version to be submitted.

\section{Conflict of Interest Disclosures}

None.

\section{Ethical Statement}

The authors have no relevant affiliations or financial involvement with any organization or entity with a financial interest in or financial conflict with the subject matter or materials discussed in the manuscript. This includes employment, consultancies, honoraria, stock ownership or options, expert testimony, grants, or patents received or pending or royalties.

\section{References}

1. Picard C, Bobby Gaspar H, Al-Herz W, Bousfiha A, Casanova JL, Chatila T, et al. International union of immunological societies: 2017 primary immunodeficiency diseases committee report on inborn errors of immunity. J Clin Immunol. 2018;38(1):96128. doi: 10.1007/s10875-017-0464-9.

2. Bazregari S, Azizi G, Tavakol M, Asgardoon MH, Kiaee F, Tavakolinia N, et al. Evaluation of infectious and non-infectious complications in patients with primary immunodeficiency. Cent Eur J Immunol. 2017;42(4):336-41. doi: 10.5114/ ceji.2017.72825.

3. Amr SS, Tbakhi A. Abu Bakr Muhammad Ibn Zakariya Al Razi (Rhazes): philosopher, physician and alchemist. Ann Saudi Med. 2007;27(4):305-7. doi: 10.5144/0256-4947.2007.305.

4. Aghamohammadi A, Moin M, Rezaei N. History of primary immunodeficiency diseases in Iran. Iran J Pediatr. 2010;20(1):16-34.

5. Abolhassani H, Rezaei N, Aghamohammadi A. Recent advances and current status of primary immunodeficiency disease in Iran. Immunol Genet J. 2018;1(1):1-33. doi: 10.22034/IGJ.2018.69304/.

6. Aghamohammadi A, Moin M, Farhoudi A, Rezaei N, Pourpak Z, Movahedi M, et al. Efficacy of intravenous immunoglobulin on the prevention of pneumonia in patients with agammaglobulinemia. FEMS Immunol Med Microbiol. 2004;40(2):113-8. doi: 10.1016/S0928-8244(03)00304-3.

7. Abolhassani H, Kiaee F, Tavakol M, Chavoshzadeh Z, Mahdaviani SA, Momen T, et al. Fourth update on the Iranian national registry of primary immunodeficiencies: Integration of molecular diagnosis. J Clin Immunol. 2018;38(7):816-32 . doi: 10.1007/s10875-018-0556-1.

8. Aghamohammadi A, Mohammadinejad P, Abolhassani $\mathrm{H}$, Mirminachi B, Movahedi M, Gharagozlou M, et al. Primary immunodeficiency disorders in Iran: update and new insights from the third report of the national registry. J Clin Immunol. 2014;34(4):478-90. doi: 10.1007/s10875-014-0001-z.

9. Aghamohammadi A, Moein M, Farhoudi A, Pourpak Z, Rezaei N, Abolmaali K, et al. Primary immunodeficiency in Iran: first report of the national registry of PID in children and adults. J Clin Immunol. 2002;22(6):375-80. doi: 10.1023/a:1020660416865.

10. Rezaei N, Aghamohammadi A, Moin M, Pourpak Z, Movahedi $M$, Gharagozlou M, et al. Frequency and clinical manifestations of patients with primary immunodeficiency disorders in Iran: update from the Iranian Primary Immunodeficiency Registry. J Clin Immunol. 2006;26(6):519-32. doi: 10.1007/s10875-0069047-x.

11. Abolhassani $H$, Mirminachi B, Daryabeigi $M$, Agharahimi Z, Aghamohammadi A, Rabbani A, et al. Evaluation of physicians' awareness of pediatric diseases in iran. Iran J Pediatr. 2014;24(1):87-92.

12. Nourijelyani K, Aghamohammadi A, Salehi Sadaghiani M, Behniafard N, Abolhassani H, Pourjabar S, et al. Physicians awareness on primary immunodeficiency disorders in Iran. Iran J Allergy Asthma Immunol. 2012;11(1):57-64.

13. Yazdani R, Abolhassani H, Kiaee F, Habibi S, Azizi G, Tavakol $M$, et al. comparison of common monogenic defects in a large predominantly antibody deficiency cohort. J Allergy Clin Immunol Pract. 2019;7(3):864-78. doi: 10.1016/j. jaip.2018.09.004.

14. Azizi G, Yazdani R, Rae W, Abolhassani H, Rojas M, Aghamohammadi A, et al. Monogenic polyautoimmunity in primary immunodeficiency diseases. Autoimmun Rev. 2018;17(10):1028-39. doi: 10.1016/j.autrev.2018.05.001.

15. Abolhassani $\mathrm{H}$, Aghamohammadi A, Fang $M$, Rezaei $N$, Jiang C, Liu X, et al. Clinical implications of systematic phenotyping and exome sequencing in patients with primary antibody deficiency. Genet Med. 2019;21(1):243-51. doi: 10.1038/ s41436-018-0012-x.

16. Klein C, Grudzien M, Appaswamy G, Germeshausen M, Sandrock I, Schaffer AA, et al. HAX1 deficiency causes autosomal recessive severe congenital neutropenia (Kostmann disease). Nat Genet. 2007;39(1):86-92. doi: 10.1038/ng1940.

17. Boztug K, Appaswamy G, Ashikov A, Schaffer AA, Salzer U, Diestelhorst J, et al. A syndrome with congenital neutropenia and mutations in G6PC3. N Engl J Med. 2009;360(1):32-43. doi: 10.1056/NEJMoa0805051.

18. Grenda DS, Murakami M, Ghatak J, Xia J, Boxer LA, Dale D, et al. Mutations of the ELA2 gene found in patients with severe congenital neutropenia induce the unfolded protein response and cellular apoptosis. Blood. 2007;110(13):4179-87. doi: 10.1182/blood-2006-11-057299.

19. Boztug K, Jarvinen PM, Salzer E, Racek T, Monch S, Garncarz W, et al. JAGN1 deficiency causes aberrant myeloid cell homeostasis and congenital neutropenia. Nat Genet. 2014;46(9):1021-7. doi: 10.1038/ng.3069.

20. Glocker EO, Hennigs A, Nabavi M, Schaffer AA, Woellner $C$, Salzer $U$, et al. A homozygous CARD9 mutation in a family with susceptibility to fungal infections. N Engl J Med. 2009;361(18):1727-35. doi: 10.1056/NEJMoa0810719.

21. Abdollahpour H, Appaswamy G, Kotlarz D, Diestelhorst J, Beier R, Schaffer AA, et al. The phenotype of human STK4 deficiency. Blood. 2012;119(15):3450-7. doi: 10.1182/ blood-2011-09-378158.

22. Lopez-Herrera G, Tampella G, Pan-Hammarstrom Q, Herholz P, Trujillo-Vargas CM, Phadwal K, et al. Deleterious mutations in LRBA are associated with a syndrome of immune deficiency and autoimmunity. Am J Hum Genet. 2012;90(6):986-1001. doi: 10.1016/j.ajhg.2012.04.015.

23. Abolhassani $H$, Edwards ES, Ikinciogullari $A$, Jing $H$, Borte $S$, Buggert $M$, et al. Combined immunodeficiency and Epstein-Barr virus-induced B cell malignancy in humans with inherited CD70 deficiency. J Exp Med. 2017;214(1):91-106. doi: 10.1084/jem.20160849.

24. Abolhassani H, Sadaghiani MS, Aghamohammadi A, Ochs HD, Rezaei N. Home-based subcutaneous immunoglobulin versus hospital-based intravenous immunoglobulin in treatment of primary antibody deficiencies: systematic review 
and meta analysis. J Clin Immunol. 2012;32(6):1180-92. doi: 10.1007/s10875-012-9720-1.

25. Abolhassani H, Sagvand BT, Shokuhfar T, Mirminachi B, Rezaei N, Aghamohammadi A. A review on guidelines for management and treatment of common variable immunodeficiency. Expert Rev Clin Immunol. 2013;9(6):56174; quiz 75. doi: 10.1586/eci.13.30.

26. Aghamohammadi A, Abolhassani $H$, Mohammadinejad $P$, Rezaei N. The approach to children with recurrent infections. Iran J Allergy Asthma Immunol. 2012;11(2):89-109. doi: 011.02/ijaai.89109.

27. Aghamohammadi A, Cheraghi T, Gharagozlou M, Movahedi M, Rezaei N, Yeganeh M, et al. IgA deficiency: Correlation between clinical and immunological phenotypes. J Clin Immunol. 2009;29(1):130-6. doi: 10.1007/s10875-0089229-9.

28. Aghamohammadi A, Farhoudi A, Moin M, Pourpak Z, Rezaei N, Nikzad $M$, et al. Adverse effects of intravenous immunoglobulin therapy in patients with antibody deficiency. Iran J Allergy Asthma Immunol. 2003;2(3):121-6. doi: 02.03/ ijaai.121126.

29. Yazdani R, Fekrvand S, Shahkarami S, Azizi G, Moazzami B, Abolhassani $\mathrm{H}$, et al. The hyper lgM syndromes: Epidemiology, pathogenesis, clinical manifestations, diagnosis and management. Clin Immunol. 2018;198:19-30. doi: 10.1016/j. clim.2018.11.007.

30. Yazdani R, Abolhassani H, Asgardoon M, Shaghaghi M, Modaresi M, Azizi $G$, et al. Infectious and noninfectious pulmonary complications in patients with primary immunodeficiency disorders. J Investig Allergol Clin Immunol. 2017;27(4):213-24. doi: 10.18176/jiaci.0166.

31. Azizi G, Ziaee V, Tavakol M, Alinia T, Yazdai R, Mohammadi $\mathrm{H}$, et al. Approach to the management of autoimmunity in primary immunodeficiency. Scand J Immunol. 2017;85(1):1329. doi: $10.1111 / \mathrm{sji} .12506$.

32. Yazdani R, Azizi G, Abolhassani H, Aghamohammadi A. Selective IgA deficiency: Epidemiology, Pathogenesis, clinical phenotype, diagnosis, prognosis and management. Scand J Immunol. 2017;85(1):3-12. doi: 10.1111/sji.12499.

33. Abolhassani $\mathrm{H}$, Rezaei $\mathrm{N}$, Mohammadinejad P, Mirminachi B, Hammarstrom L, Aghamohammadi A. Important differences in the diagnostic spectrum of primary immunodeficiency in adults versus children. Expert Rev Clin Immunol. 2015;11(2):289-302. doi: 10.1586/1744666X.2015.990440.

34. Abolhassani $\mathrm{H}$, Parvaneh $\mathrm{N}$, Rezaei N, Hammarstrom L, Aghamohammadi A. Genetic defects in B-cell development and their clinical consequences. J Investig Allergol Clin Immunol. 2014;24(1):6-22.

35. Azizi G, Ahmadi M, Abolhassani H, Yazdani R, Mohammadi $\mathrm{H}$, Mirshafiey A, et al. Autoimmunity in primary antibody deficiencies. Int Arch Allergy Immunol. 2016;171(3-4):18093. doi: 10.1159/000453263.

36. Arandi N, Mirshafiey A, Jeddi-Tehrani M, Shaghaghi M, Sadeghi B, Abolhassani $\mathrm{H}$, et al. Alteration in frequency and function of $\mathrm{CD} 4(+) \mathrm{CD} 25(+) \mathrm{FOXP} 3(+)$ regulatory T cells in patients with immune thrombocytopenic purpura. Iran J Allergy Asthma Immunol. 2014;13(2):85-92.

37. Fang $M$, Abolhassani $H$, Lim $C K$, Zhang J, Hammarstrom L. Next generation sequencing data analysis in primary immunodeficiency disorders - future directions. J Clin Immunol. 2016;36 Suppl 1:68-75. doi: 10.1007/s10875-0160260-y.

38. Maccari ME, Abolhassani $H$, Aghamohammadi A, Aiuti A, Aleinikova O, Bangs $C$, et al. Disease evolution and response to rapamycin in activated phosphoinositide 3-kinase delta syndrome: the european society for immunodeficienciesactivated phosphoinositide 3-kinase delta syndrome registry. Front Immunol. 2018;9:543. doi: 10.3389/fimmu.2018.00543.
39. Azizi G, Abolhassani $H$, Yazdani R, Mohammadikhajehdehi S, Parvaneh N, Negahdari B, et al. New therapeutic approach by sirolimus for enteropathy treatment in patients with LRBA deficiency. Eur Ann Allergy Clin Immunol. 2017;49(5):235-9. doi: 10.23822/EurAnnACl.1764-1489.22.

40. Alkhairy OK, Abolhassani H, Rezaei N, Fang M, Andersen KK, Chavoshzadeh Z, et al. Spectrum of phenotypes associated with mutations in LRBA. J Clin Immunol. 2016;36(1):33-45. doi: 10.1007/s10875-015-0224-7.

41. Yousefzadegan S, Tavakol M, Abolhassani H, Nadjafi A, Mansouri S, Yazdani R, et al. Systematic investigation for underlying causes of recurrent infections in children: surveillance of primary immunodeficiency. Eur Ann Allergy Clin Immunol. 2018;50(2):72-80. doi: 10.23822/ EurAnnACl.1764-1489.39.

42. Shaghaghi $M$, Irannejad $M$, Abolhassani $H$, Shahmahmoodi S, Hamidieh AA, Soleyman-Jahi S, et al. Clearing vaccinederived poliovirus infection following hematopoietic stem cell transplantation: a Case report and review of literature. J Clin Immunol. 2018;38(5):610-6. doi: 10.1007/s10875-0180521-z.

43. Shaghaghi $M$, Soleyman-Jahi $S$, Abolhassani H, Yazdani R, Azizi G, Rezaei N, et al. New insights into physiopathology of immunodeficiency-associated vaccine-derived poliovirus infection; systematic review of over 5 decades of data. Vaccine. 2018;36(13):1711-9. doi: 10.1016/j.vaccine.2018.02.059.

44. Aghamohammadi A, Abolhassani $\mathrm{H}$, Kutukculer N, Wassilak SG, Pallansch MA, Kluglein S, et al. Patients with primary immunodeficiencies are a reservoir of poliovirus and a risk to polio eradication. Front Immunol. 2017;8:685. doi: 10.3389/ fimmu.2017.00685.

45. Yazdani R, Abolhassani H, Tafaroji J, Azizi G, Hamidieh AA, Chou J, et al. Cernunnos deficiency associated with BCG adenitis and autoimmunity: First case from the national Iranian registry and review of the literature. Clin Immunol. 2017;183(201-6. doi: 10.1016/j.clim.2017.07.007.

46. Shaghaghi M, Shahmahmoodi S, Abolhassani H, SoleymanJahi S, Parvaneh L, Mahmoudi S, et al. Vaccine-derived polioviruses and children with primary immunodeficiency, Iran, 1995-2014. Emerg Infect Dis. 2016;22(10):1712-9. doi: 10.3201/eid2210.151071.

47. Aghamohammadi A, Bahrami A, Mamishi S, Mohammadi B, Abolhassani H, Parvaneh N, et al. Impact of delayed diagnosis in children with primary antibody deficiencies. J Microbiol Immunol Infect. 2011;44(3):229-34. doi: 10.1016/j. jmii.2011.01.026.

48. Abolhassani H, Wang N, Aghamohammadi A, Rezaei N, Lee $\mathrm{YN}$, Frugoni $\mathrm{F}$, et al. A hypomorphic recombination-activating gene 1 (RAG1) mutation resulting in a phenotype resembling common variable immunodeficiency. J Allergy Clin Immunol. 2014;134(6):1375-80. doi: 10.1016/j.jaci.2014.04.042.

49. Mohammadinejad $\mathrm{P}$, Aghamohammadi $\mathrm{A}$, Abolhassani $\mathrm{H}$, Sadaghiani MS, Abdollahzade S, Sadeghi B, et al. Pediatric patients with common variable immunodeficiency: long-term follow-up. J Investig Allergol Clin Immunol. 2012;22(3):20814

50. Abolhassani $\mathrm{H}$, Chou J, Bainter W, Platt CD, Tavassoli $\mathrm{M}$, Momen $\mathrm{T}$, et al. Clinical, immunologic, and genetic spectrum of 696 patients with combined immunodeficiency. J Allergy Clin Immunol. 2018;141(4):1450-8. doi: 10.1016/j. jaci.2017.06.049.

51. Alkhairy OK, Rezaei N, Graham RR, Abolhassani H, Borte S, Hultenby K, et al. RAC2 loss-of-function mutation in 2 siblings with characteristics of common variable immunodeficiency. J Allergy Clin Immunol. 2015;135(5):1380-4 e1-5. doi: 10.1016/j.jaci.2014.10.039.

52. Aghamohammadi A, Abolhassani H, Biglari M, Abolmaali S, Moazzami K, Tabatabaeiyan M, et al. Analysis of switched 
memory B cells in patients with IgA deficiency. Int Arch Allergy Immunol. 2011;156(4):462-8. doi: 10.1159/000323903.

53. Yazdani R, Fatholahi M, Ganjalikhani-Hakemi M, Abolhassani $\mathrm{H}$, Azizi G, Hamid KM, et al. Role of apoptosis in common variable immunodeficiency and selective immunoglobulin A deficiency. Mol Immunol. 2016;71:1-9. doi: 10.1016/j. molimm.2015.12.016.

54. Azizi G, Abolhassani $\mathrm{H}$, Asgardoon $\mathrm{MH}$, Rahnavard J, Dizaji $M Z$, Yazdani R, et al. The use of immunoglobulin therapy in primary immunodeficiency diseases. Endocr Metab Immune Disord Drug Targets. 2016;16(2):80-8. doi: 10.2174/1871530 316666160724214418.

55. Azizi G, Abolhassani $H$, Asgardoon $M H$, Shaghaghi $S$, Negahdari B, Mohammadi J, et al. Managing patients with side effects and adverse events to immunoglobulin therapy. Expert Rev Clin Pharmacol. 2016;9(1):91-102. doi: 10.1586/17512433.2016.1105131.

56. Abolhassani $\mathrm{H}$, Asgardoon $\mathrm{MH}$, Rezaei N, Hammarstrom L, Aghamohammadi A. Different brands of intravenous immunoglobulin for primary immunodeficiencies: how to choose the best option for the patient? Expert Rev Clin Immunol. 2015;11(11):1229-43. doi: 10.1586/1744666X.2015.1079485.

57. Abolhassani H, Aghamohammadi A, Hammarstrom L. Monogenic mutations associated with IgA deficiency. Expert Rev Clin Immunol. 2016;12(12):1321-35. doi:
10.1080/1744666X.2016.1198696.

58. Abolhassani H, Aghamohammadi A, Abolhassani F, Eftekhar $\mathrm{H}$, Heidarnia M, Rezaei N. Health policy for common variable immunodeficiency: burden of the disease. J Investig Allergol Clin Immunol. 2011;21(6):454-8.

59. Gholami K, Laali E, Abolhassani H, Ahmadvand A, Mohebbi $\mathrm{N}$, Javadi MR, et al. Costs of Hospital Admission on Primary Immunodeficiency Diseases. Iran J Public Health. 2017;46(3):342-50.

60. Sadeghi B, Abolhassani H, Naseri A, Rezaei N, Aghamohammadi A. Economic burden of common variable immunodeficiency: annual cost of disease. Expert Rev Clin Immunol. 2015;11(5):681-8. doi: 10.1586/1744666X.2015.1029457.

61. Abolhassani H, Aghamohammadi A, Pourjabbar S, Salehi Sadaghiani M, Nikayin S, Rabiee A, et al. Psychiatric aspects of primary immunodeficiency diseases: the parental study. Iran J Allergy Asthma Immunol. 2013;12(2):176-81.

62. Aghamohammadi A, Montazeri A, Abolhassani H, Saroukhani S, Pourjabbar S, Tavassoli M, et al. Health-related quality of life in primary antibody deficiency. Iran J Allergy Asthma Immunol. 2011;10(1):47-51. doi: 010.01/ijaai.4751.

63. Ataeinia B, Montazeri A, Tavakol M, Azizi G, Kiaee F, Tavakolinia N, et al. Measurement of health-related quality of life in primary antibody-deficient patients. Immunol Invest. 2017; 46(4):329-40. doi: 10.1080/08820139.2016.1258710. 